\title{
General Guiding Center Drifts in TRANSP
}

by

\author{
R. T. McCann*, R. J. Goldston, and D. C. McCune \\ Princeton Plasma Physics Laboratory \\ Princeton, N.J. 08543
}

*John von Neumann National Supercomputer Center

PPPL--2684

DE90 007947

\section{Introduction}

This document describes the guiding center drift equations and some of the numerical techniques used in the Princeton Plasma Physics Laboratory's transport analysis code TR.ANSP which is used to evaluate the effect of neutral beam heating on various tokamak experiments at PPPL and elsewhere. The equations used are adapted from White ${ }^{1}$ and Boozer ${ }^{2}$ and are specialized to two dimensions by assuming perfect toroidal symmetry. The resulting minor cross section of the plasma is almost coplanar. Deviations from being coplanar are ignored.

\section{DISCLAIMER}

This report was prepared as an account of work sponsored by an agency of the United States Government. Neither the United States Government nor any agency thercor, nor any of their employes, makes any warranty, express or implied, or assumes any legal liability or responsibility for the accuracy, completeress, or usefulness of any information, apparatus, product, or process disclosed, or iepresents that its use would not infringe privately owped rights. Reference herein to any specific commercial product, process, or service by trade name, tradematk, manufacturer, or otherwise does not necessarily constitute or imply its endorsement, recommendation, of favoring by the United States Government or any agency thereof. The views and opinions of authors expressed herein do not necessarily state or reflect those of the United States Government or any agency thereof. 


\section{Guiding Center Drift Equations}

In order to derive the guiding center drift equations. it is useful to consider either the Lagrangian or the Hamiltonian for a magnetic dipole in an electro-magnetic field. Rather than using the exact Hamiltonain or Lagrangian, we follow Boozer and keep only the energy due to the motion of the dipole parallel to the magnetic field, the energy due to the magnetic moment (the gyro-kinetic energy), and the potential energy due to the electric field. Heuristically,

$$
H=\frac{1}{2} m v_{\|}^{2}+\mu B+e \Phi
$$

and

$$
L=\frac{1}{2} m v_{\|}^{2}+(e / c) \mathbf{A} \cdot \mathbf{v}-\mu B-e \Phi
$$

where $\Phi$ is the electric potential, $B=\|\mathbf{B}\|, \mathbf{B}$ is the magnetic field, $\mathbf{A}$ is the magnetic vector potential, $V_{\|}$is the velocity parallel to the magnetic field, and $\mathbf{v}$ is the velocity of the guiding center relative to the plasma.

In order to express the equations of motion in magnetic coordinates, it is first necessary to express $H$ and $L$ in that form. This may be accomplished by expressing the magnetic field in magnetic coordinates:

$$
\mathbf{B}=\nabla \Psi_{T} \times \nabla \theta+\nabla \phi \times \nabla \Psi_{P}\left(\Psi_{T}\right)
$$

and

$$
\mathbf{B}=g\left(\Psi_{T}\right) \nabla \phi+I\left(\Psi_{T}\right) \nabla \theta+\delta\left(\Psi_{T}, \theta, \phi\right) \nabla \Psi_{T} .
$$

A vector potential for the magnetic field is

$$
\mathbf{A}=\Psi_{T} \nabla \theta-\Psi_{P} \nabla \phi .
$$


Where $\Psi_{T}$ is toroidal flux, $\Psi_{P}$ is poloidal flux, $\theta$ is a poloidal angle. and $\phi$ is a toroidal angle. With some work it can be shown that

$$
V_{\|}=(g \dot{\phi}+I \dot{\theta}) / B
$$

With these things in hand, the Lagrangian can be expressed as

$$
L=\frac{1}{2} m(g \dot{\phi}+I \dot{\theta})^{2} / B^{2}+(e / c)\left(\Psi_{T} \dot{\theta}-\Psi_{P} \dot{\varphi}\right)-\mu B-e \Phi
$$

From this one can then generate the Hamiltonian in the usual way, noting that $\dot{\Psi}_{P}$ is missing from the Lagrangian:

$$
H=P_{\theta} \dot{\theta}+P_{\phi} \dot{\phi}-L
$$

Then the equations of motion follow either from the Euler equations or from Hamilton's equations. A similar derivation is given in detail by Chancs and White. ${ }^{1}$ 


\section{A Novel Derivation of the Alfvén Drift Equations}

It is instructive to note that, by starting with the Lagrangian in general form and by expanding in gyro radius to system size, one can derive the usual Alfvén drift velocity. First let

$$
\mathrm{V}=V_{\|} \hat{\mathrm{b}}+\mathrm{V}_{\perp} \text { : }
$$

then expand each of these velocities as well as the time derivatives order by order:

$$
\begin{aligned}
V_{\|} & =V_{\| 0}+V_{\| 1}+V_{\| 2}+\cdots \\
\mathrm{V}_{\perp} & =V_{\perp 0}+V_{\perp 1}+V_{\perp 2}+\cdots \\
\frac{\partial}{\partial t} & =\frac{\partial}{\partial t_{0}}+\frac{\partial}{\partial t_{1}}+\frac{\partial}{\partial t_{2}}+\cdots
\end{aligned}
$$

One should then identify the lowest order time scale as

$$
\frac{\partial}{\partial t_{0}} \sim V_{0} / L
$$

and then one must give the expansion parameter

$$
\rho / L=V_{0} / L \Omega_{c i}
$$

By asserting that the $E \times B$ drift velocity is first order

$$
V_{E \times B} \sim V_{1},
$$

one discovers that

$$
\frac{c \Phi}{L B} \sim \frac{V_{0} \rho}{L} \sim \frac{m v_{0}^{2} c}{L e B}
$$


or

$$
e \Phi \sim m V_{0}^{-2} \sim \mu B
$$

The result of the expansion through zeroth order is as follows:

$$
\mathrm{V}_{\perp 0}=0
$$

so

$$
\mathbf{V}_{0}=V_{110} \hat{b}
$$

and

$$
m \frac{\partial V_{\| 0}}{\partial t_{0}}=-\hat{\mathbf{b}} \cdot \mu \nabla B
$$

The first order terms give the familiar cross field drifts,

$$
\frac{e}{c}\left(\mathbf{V}_{\perp 1}-\mathbf{V}_{\mathbf{E} \times \mathbf{B}}\right) B^{2}=\mu \mathrm{B} \times \nabla B+m V_{\| 1}^{2} \mathbf{B} \times(\hat{\mathbf{b}} \cdot \nabla) \hat{\mathbf{b}}
$$

and

$$
\left(\frac{\partial V_{\|}}{\partial t}\right)_{1}=-\frac{c m}{e B^{2}} V_{\| 0}^{2}(\nabla \times \mathbf{B})_{\perp} \cdot \frac{\mu}{m V_{\| 0}} \nabla B-V_{\| 0} \frac{\partial \ln (B J)}{\partial t}
$$




\section{Momentum and Energy Conserving Drifts}

In order to assure closed drift orbits, it is necessary to have at least two conserved quantities in addition to the magnetic moment of the guiding centers. In our case, we would like to have the particle energy and canonical angular womentum conserved. This is possible in our case since we advance the drift equations assuming a fixed geometry. In this case the Alfvén drifts conserve energy, but are not Hamiltonian and do not conserve canonical angular momentum. We address this by adding a snall term (third order in parallel gyroradius over minor radius and proportional to the parallel component of plasma current) to the usual drifts to give us equations which conserve both energy and a convenient "momentum." Hence we end up with closed drift orbits derived from a Hamiltonian which is correct to second order in parallel gyroradius divided by plasma minor radius.

Using conservation of energy we are able to solve for a new parallel drift velocity after advancing the guiding center. This gives us analytically conserved momentum, and numerical conservation to a few parts in a million. Then we hold the particle momentum constant and change the geometry whe' neeled, giving a new particle energy.

Including the drift due to a noninductive electric field, the momentum conserving drift velocity is

$$
\mathrm{V}=-\frac{c}{e} \frac{\nabla A \times \mathrm{B}}{B^{2}}+V_{\|} \hat{\mathrm{b}}-\frac{c}{e} \frac{m V_{\|}^{2}}{B} \frac{\nabla B \times \mathrm{B}}{B^{2}} \div \frac{c}{\epsilon} \cdot n V_{\|}^{2} \frac{\nabla \times \mathrm{B}}{B^{2}}
$$

where

$$
A=\mu B+e \Phi,
$$

where $\Phi$ is the radial electric potential expressed as a function of toroidal flux and $B$ is the magnitude of the magnetic field 
expressed as a function of both toroidal flux and poloidal angle. To conver this to magnetic coordinates, we use the following definitions:

$$
\begin{aligned}
\mathbf{B} & =g \nabla \zeta+I \nabla \theta+\delta \nabla \Psi_{P}, \\
J^{-1} & =\nabla \Psi_{P} \times \nabla \theta \cdot \nabla \zeta, \\
\frac{d \Psi_{P}}{d t} & =\mathrm{V} \cdot \nabla \Psi_{P}, \\
\frac{d \theta}{d t} & =\mathrm{V} \cdot \nabla \theta
\end{aligned}
$$

where we have taken

$$
\frac{\partial}{\partial t}=0
$$

so that

$$
\frac{d}{d t} \equiv \mathrm{V} \cdot \nabla
$$

This means that we are assuming constant magnetic geometry during the orbit integration step. The effects of changing geometry are handled separately in a compression subroutine. Since the geometry is usually either constant or slowly changing, this eliminates considerable complexity in the orbit subroutines. Also note that we have taken $\nabla \zeta= \pm \nabla \phi$ to allow the coordinate system to be either right or left handed as needed to deal with the possibility that the toroidal components of the ohmic heating current and the magnetic field can be eitiner parallel or antiparallel.

The above defin.tions give the equations for the guiding 
center drifts in magnetic coordinates:

$$
\begin{aligned}
\frac{d \Psi_{P}}{d t}= & -\frac{g c}{J B^{2}} \frac{\partial \Phi}{\partial \theta}+0 \\
& -\frac{g c}{e J B^{2}}\left(\mu+\frac{m V_{\|}^{2}}{B}\right) \frac{\partial B}{\partial \theta}+0 \\
\frac{d \theta}{d t}= & +\frac{g c}{J B^{2}} \frac{\partial \Phi}{\partial \Psi_{P}}+\frac{V_{\|}}{J B} \\
& +\frac{g c}{e J B^{2}}\left(\mu+\frac{m V_{\|}^{2}}{B}\right) \frac{\partial B}{\partial \Psi_{P}}-\frac{c}{e J B^{2}} m V_{\|}^{2} \frac{\partial g}{\partial \Psi_{P}}
\end{aligned}
$$

and

$$
\frac{d V_{\|}}{d t}=\left(-\frac{\mu}{m J B}+\frac{\mu c V_{\|}}{e J B^{2}} \frac{\partial g}{\partial \Psi_{P}}+\frac{g c V_{\|}}{J B^{3}} \frac{\partial \Phi}{\partial \Psi_{P}}\right) \frac{\partial B}{\partial \theta} .
$$




\section{Practical Representation of Magnetic Geometry}

From a practical point of view, it is convenient to express $J$ in terms of the cylindrical coordinates $R$ and $Y$ where $R$ is the horizontal radial distance from the symmetry axis of the tokamak and where $Y$ is the distance above the midplane:

$$
J=R\left(\frac{\partial R}{\partial \Psi_{P}} \frac{\partial Y}{\partial \theta}-\frac{\partial R}{\partial \theta} \frac{\partial Y}{\partial \Psi_{P}}\right)
$$

or, in terms of $\xi$,

$$
J=R \frac{\partial \xi}{\partial \Psi_{P}}\left(\frac{\partial R}{\partial \xi} \frac{\partial Y}{\partial \theta}-\frac{\partial R}{\partial \theta} \frac{\partial Y}{\partial \xi}\right)
$$

where

$$
\xi \equiv \sqrt{\Psi_{T} / \Psi_{T}^{\operatorname{Lim}}}
$$

Thus $\xi$ varies from zero on the magnetic axis to one on the plasma surface. One should note that the third direction, $\hat{\mathbf{Z}}$, is in the direction $\hat{\mathbf{R}} \times \hat{\mathbf{Y}}$, forming a right handed, orthozonal coordinate system. $(\xi, \theta, \phi)$ also forms a right handed coordi. nate system. However, $\left(\Psi_{P}, \theta, \zeta\right)$ can be either right handed or leit handed as needed in order to assure that $\hat{\zeta} \cdot \mathbf{B}$ is positive. This usage of potentially left handed coordinc tes is retained because, in tokamak parlance, the parallel direction refers to the direction the toroidal component of the plasma ohmic heating current flows, and that can be either parallel or antiparallel to the toroidal component of the magnetic field.

In order to nininize computation, the MHD equilibrium is specified in terms of a Fourier-spline representation for $R$ and $Y$ :

$$
\begin{aligned}
& R=\sum R_{j}(\xi) \cos (j \theta) \\
& Y=\sum Y_{j}(\xi) \sin (j \theta)
\end{aligned}
$$


where the coefficients $R_{j}(\xi)$ and $Y_{j}(\xi)$ are functions of $\xi$ which are represented as cubic splines. Further, the definition of $\theta$ has been adjusted so that the Fourier coefficients display the maximum convergence rate on each flux surface. ${ }^{3.4 .5}$ That minimizes the number of moments which must be retained to obtain a given accuracy in representing a given flux surface.

It has been found that this is more efficient than choosing $\theta$ such that the Jacobian is proportional to a function of $\xi$ times a power of $B(\xi, \theta)$. The reason is that, with the simplified Jacobian approach, the number of moments needed in order to describe the flux surfaces with equal accuracy is more than twice as many as in the "scrunched moments" approach, so that evaluating twice as many Fourier spline functions still results in less work overall.

\section{MHD Equilibrium Solution}

The Fourier coefficients for $R$ and $Y$ can be computed in two ways in TRANSP. In both cases, experimental measurements provide the shape of the $\xi=1$ surface which is then supplied to a routine which solves for the shape of the interior flux surfaces. Both methods are due to Hirshman ${ }^{5.6}$. The first, VMOMS $^{6}$, uses a shooting method to solve a set of couple. ordinary differential equations for a related set of Fourier coefficients which are then mapped into the set used in TRANSP. In this case, the equations represent a flux surface average of the magnetic force balance. In the second case, VMEC 5 , the method of steepest descent is used to solve the full GradShafranov equation, and the resulting geometry is mapped into the TRANSP Fourier-spline representation.

The flux surfaces exterior to the plasma are extrapolated in an ad hoc way which allows the Fourier-spline coefficients 
to be continuous across the plasma surface without creating an X-point outside the plasma (even though oine may exist). In fact, the exterior surfaces asymptotically approach circular shape.

The exterior field is in no way intended to act as a valid solution to Maxwell's equations in the vacuum region. Instead, the purpose of the exterior representation is to allow ions and neutral particles to dift out of the plasma and to interact with solid objects such as limiters, diagnostic equipment, and support structure. A fully valid solution to Maxwell's equations outside the plasma would require too much effort on the part of the experimenters to deal with all the eddy currents in the myriad objects near the vacuum vessel. Being a fully three dimensional calculation, it would also be computationally expensive. Such modeling of the vacuum and scrape off regions is beyond the purpose of TRANSP at this time. 


\section{Usefuì Trarsformations}

The magnetic field is more efficiently stored in Fourierspline rep sentation as $1 / B$ since this is nearly proportional to $R$ which has only low order Fourier terms. In order to make best use of this, the orbit equations are recast i.l. terms of

$$
W \equiv 1 / B
$$

noring that

$$
\frac{\partial W}{\partial s}=-\left(\frac{1}{B^{2}}\right) \frac{\partial B}{\partial s}
$$

where $s$ is any parameter. Hence, the orbit equations become

$$
\begin{aligned}
\frac{d \Psi_{P}}{d t}= & -\frac{g c W^{2}}{J} \frac{\partial \Phi}{\partial \theta}+0 \\
& +\frac{g c}{e J}\left(\mu+m W V_{\|}^{2}\right) \frac{\partial W}{\partial \theta}+0 \\
\frac{d \theta}{d t}= & +\frac{g c W^{2}}{J} \frac{\partial \Phi}{\partial \Psi_{P}}+\frac{W V_{\|}}{J} \\
& -\frac{g c}{e J}\left(\mu+m W V_{\|}^{2}\right) \frac{\partial W}{\partial \Psi_{P}}-\frac{c W^{2}}{e J} m V_{\|}^{2} \frac{\partial g}{\partial \Psi_{P}},
\end{aligned}
$$

and

$$
\frac{d V_{\|}}{d t}=\left(+\frac{\mu}{m J W}-\frac{\mu c V_{\|}}{e J} \frac{\partial g}{\partial \Psi_{P}}-\frac{g c W V_{\|}}{J} \frac{\partial \Phi}{\partial \Psi_{P}}\right) \frac{\partial W}{\partial \theta} .
$$

These equations are further transformed in TRANSP so that we deal with

$$
\xi \equiv \sqrt{\Psi_{T} / \bar{\Psi}_{T}^{L i n}}
$$


Remembering that

$$
q \equiv \frac{\partial \bar{\Psi}_{T}}{\partial \Psi_{P}}
$$

we have the relationship

$$
\frac{\partial \xi}{\partial \Psi_{P}}=\frac{q}{2 \xi \Psi_{T}^{L i m}}
$$

At this point we may as well onit the $\theta$ dependence of $\Phi$. So the drift equations now become

$$
\begin{aligned}
\frac{d \xi}{d t}=0 & +0 \\
& +\frac{\partial \xi}{\partial \Psi_{P}} \frac{g c}{e J}\left(\mu+m W V_{\|}^{2}\right) \frac{\partial W}{\partial \theta}+0 \\
\frac{d \theta}{d t}= & +\frac{\partial \xi}{\partial \Psi_{P}} \frac{g c W^{2}}{J} \frac{\partial \Phi}{\partial \xi}+\frac{W V_{\|}}{J} \\
& -\frac{\partial \xi}{\partial \Psi_{P}} \frac{g c}{e J}\left(\mu+m W V_{\|}^{2}\right) \frac{\partial W}{\partial \xi}-\frac{\partial \xi}{\partial \Psi_{P}} \frac{c W^{2}}{e J} m V_{\|}^{2} \frac{\partial g}{\partial \xi}
\end{aligned}
$$

and

$$
\frac{d V_{\|}}{d_{i}}=\left(+\frac{\mu}{m J W}-\frac{\partial \xi}{\partial \Psi_{P}} \frac{\mu c V_{\|}}{e J} \frac{\partial g}{\partial \xi}-\frac{\partial \xi}{\partial \Psi_{P}} \frac{g c W V_{\|}}{J} \frac{\partial \Phi}{\partial \xi}\right) \frac{\partial W}{\partial \theta} .
$$




\section{Conserved Quantities}

The conserved quantities are magnetic moment, energy, and a quantity which is essentially the canonical angular momentum. These are defined as follows:

$$
\begin{gathered}
\mu \equiv \frac{m V_{\perp}^{2}}{2 B} \\
E \equiv \frac{1}{2} m V_{\|}^{2}+\mu B+e \Phi
\end{gathered}
$$

and

$$
P_{\phi} \equiv m V_{\|} \frac{g}{B}-\frac{e}{c} \Psi_{P}
$$

That the energy is conserved follows directly from the lerivation of the equation for $V_{\|}$. That equation was derived by setting the time derivative of $E$ equal to zero, where

$$
\frac{d E}{d t} \equiv 0
$$

or

$$
m V_{\|} \frac{d V_{\|}}{d t}=-\mathrm{V} \cdot \nabla(\mu B+e \Phi)
$$

The conservation of $P_{\phi}$ follows from applying the same exercise to angular momentum along with direct substitution of the equation for $d V_{\|} / d t$.

That there are two conserved quantities involving $V_{\|}$allows a choice of three ways to solve for the change in $V_{\|}$: integrate $d V_{\|} / d t$, use conservation of energy, or use conservation of canonical angular momentum. Each method has its own advantages and disadvantages. Using conservation of canonical angular momentum is the simplest method, however there is 
a desire to have more accuracy in energy than in momentum. Integrating $d V_{\|} / d t$ is next in simplicity, but does not conserve either momentum or energy exactly. Finally, using conservation of energy requires the most work and requires considerable numerical sophistication, but it does provide exact conservation of energy almost everywhere.

\section{Numerical Integration Method}

The equations for $d \xi / d t$ and $d \theta / d t$ are integrated by a direct, explicit method assuming that the driving terms are time independent. Explicit tine dependence is handled elsewhere. Both equations have the form

$$
\frac{d f}{d t}=G(\xi, \theta)
$$

which is integrated as follows:

$$
f_{1}=f_{0}+\delta t G\left(\xi_{0}, \theta_{0}\right)+\frac{1}{2} \delta t^{2} \mathbf{V} \cdot \nabla G\left(\xi_{0}, \theta_{0}\right)
$$

The error terms will be proportional to $\delta t^{3}$. They are nut always small. 
Numerical Considerations in $E$ and $P_{\phi}$ Conservation

It is useful to note ai this point that, although the position errors are proportional to $\delta t^{3}$, energy and momentum errors are not always small, nor do they always scale as $\delta t^{3}$.

It has been found that it is much easier to get well behaved energy conservation if $\delta V_{\|}$is calculated from momentum conservation than it is to get well behaved momentum conservation by computing $\delta V_{\|}$from $\delta E=0$. In fact, for reasonable choices for $\delta t, \delta E$ is proportional to $\delta t^{3}$ for most of the orbit.

There are exceptions for various locations. On the midplane, $\delta E$ is proportional to $\delta t^{4}$. In a PBX equilibrium which has indented flux surfaces, the $\cos (3 \theta)$ terms are important near $\theta=\pi / 6$ and $\theta=2 \pi / 3$. The orbit equations do not contain the third derivatives of the magnetic field, so at these locations field representation errors dominate the energy error; and exponential scaling of the error breaks down. In addition, near any $\theta$ where $\delta E$ changes sign, the scaling with $\delta t$ breaks down, but this is of little concern, since $\delta E$ goes through zero at those locations. 


\section{Exact Energy Conservation}

In order to achieve numerically exact energy conservation, it is best that the following should be solved for $\delta V_{\|}$:

$$
\delta V_{\|}^{2}+2.0 V_{\|} \delta V_{\|}+\frac{2}{m}(\mu \delta B+e \delta \Phi)=0 .
$$

This minimizes the loss of precision due to taking the difference of two very sinilar large numbers in order to compute a small number. Newton's numerically optimum expression for the roots of the quadratic equation is used in order to get full accuracy, and the root closest to that computed from the equation for $d V_{\|} / d t$ is used.

If

$$
A x^{2}+B x+C=0
$$

then

$$
\begin{aligned}
& x_{1}=-\frac{2 C \operatorname{Sgn}(B)}{|B|+\sqrt{B^{2}-4 A C}} \\
& x_{2}=-\frac{\operatorname{Sgn}(B)\left(|B|+\sqrt{B^{2}-4 A C}\right)}{2 A}
\end{aligned}
$$

where both roots are well conditioned as $A C \longrightarrow 0$. The case $B=C=0$ is a double root at $x=0$ which must be handled separately. The expression for $x_{\mathrm{i}}$ was derived by noticing that $x_{2}$ is well conditioned and by using the fact that the product of the two roots of the quadratic equation is always $C / A$. So,

$$
x_{1}=(C / A) / x_{2} .
$$

A similar procedure, using products of roots, will give well condicioned roots for the cubic and quartic equations as well. 
In addition, in order to get full machine precision, it is necessary to accumulate to double precision all quantities from which square roots are to be extracted. Specifically; the discriminant in the quadratic equation above must be computed to double precision in order that the resulting square root be accurate to single precision. Similarly, when computing $V$ from $V_{\|}$and $V_{\perp}$, it is necessary to compute in double precision before taking the square root. Actually, only a few extra bits of precision are required, but not all arithmetic processors provide these guard bits, so the double precision becomes necessary for the code to be portable.

Further, it is necessary to consider the case that the discriminant is negative due to position errors resulting from integrating $d \xi / d t$ and $d \theta / d t$. In this case, $\delta V_{\|}$is obtained by integrating $d V_{\|} / d t$. Finally, it is necessary to compute a relative error in momentum. The denominator cannot be the total conserved momentum since that can be zero. The angular momentum the ion would have if it were to become nelit:alized is a reasonable choice, but each researcher will favor a different denominator. 


\section{Exact Momentum Conservation}

The following expression results in exact momentum conservation when used to compute $V_{\|}$at the end of a time step:

$$
V_{\|_{2}}=\frac{\left(m g_{1} W_{1} V_{\|_{1}}+(e / c) \delta \Psi_{P}\right)}{\left(m g_{2} W_{2}\right)}
$$

This equation is well behaved in that it always gives a $V_{\|}$consistent with the new location. For purposes of time step control. the change in energy can be evaluated as follows:

$$
\delta E=\frac{1}{2} m \delta V_{\|}^{2}+m V_{\|} \delta V_{\|}+(\mu \delta B+e \delta \Phi) .
$$

The relative error in energy is obtained by dividing by the total conserved energy of the ion. Note that no double precision is required in order to get a single precision result. 


\section{Time Step Control}

In order to minimize unnecessary computation and maximize the resulting accuracy, it is necessary to have some kind of self adjusting time step during the numerical integration of $d \xi / d t$ and $d \theta / d t$. Again there are several choices:

1. Use momentum conservation to pick $V_{\|}$and then use the error in energy relative to what is allowable in order to adjust the time step.

2. Use energy conservation to pick $V_{\|}$and then use the error in momentum conservation to adjust the time step.

3. Integrate $d V_{\|} / d t$ and use the errors in both energy and momentum to adjust the time step.

4. Pick a time step inversely proportional to the curvature of the orbit in the R-Y plane.

All of the above have serious problems. The first three have too many special cases where the method fails, and the fourth tends to spend too much time resolving the tips of banana orbits.

Features which are significantly smaller than the gyroradius are irrelevant to this simulation. What matters is that the orbits close and conserve energy, momentum, and magnetic moment in the absence of collisions and other nonideal effects. In principle, we do not care what shape the banana tip has below, roughly, the size of an ion gyro-orbit radius, so long as the ion spends a physically reasonable amount of time there. The empirical method gives reasonable looking orbits without wasting time resolving unneeded structure. The formula we use for the time step is also very simple and explicit compared to any of the other options considered,

$$
d t=0.5 \times F u d g e(\xi) \times d t_{0} \times \frac{V_{0}}{V} \times\left(1.0+3.0 \times \frac{V_{\perp}^{2}}{V^{2}}\right),
$$


where $V$ is the ion velocity including the gyromotion, $V_{\perp}$ is the ion gyrovelocity, computed from $\mu$ and $B . d t_{0} \times V_{0}$ is an arbitrarily adjustable constant, and $F u d g e(\xi)$ is equal to the plasma $q$ for $\xi \leq 0.8$ and is equal to 0.5 for $\xi>0.8$. Even with all of this care, the ratio of execution time for the orbit equations for a typical PBX bearn case to that of a typical TFTR beam case is 5 to 1 ! So we must pick our PBX cases and our TFTR compression cases with care since we cannot run very many in a week's time.

The empirical time step control formula has one free parameter' which can be adjusted via the input data file. Each different magnetic geometry, e.g., TFTR and PBX, will need a different setting of this free parameter. The method used in setting this parameter is to turn off all nonideal effects, to plot a bunch of difficult orbits such as "the wurld's fattest banana", and to adjust the parameter until 50 orbits overlay to the resolution of the plotted line width. 


\section{Alfvén Drifts in Shifted Circular Geometry}

The equations for drifts in general geometry can be simplified greatly if some knowledge of the geometry is used. If the MHD equilibrium is taken to consist of circular fiux surfaces of varying radii whose centers are shifted with respect to the center of the outermost flux surface, the equations greatly simplify since the explicit forms for $B, J$, etc. are known:

$$
\begin{aligned}
R(\xi, \theta) & =R_{0}(\xi)+A \xi \cos (\theta) \\
Y(\xi, \theta) & =A \xi \sin (\theta) \\
W(\xi, \theta) & =R(\xi, \theta) / g \\
J(\xi, \theta) & =R(\xi, \theta)\left(A+\frac{\partial R_{0}}{\partial \xi}\right) \frac{\partial \xi}{\partial \Psi_{P}}
\end{aligned}
$$

where

$$
\frac{\partial g}{\partial \xi} \equiv 0 .
$$

This leads to much simplified orbit equations:

$$
\begin{aligned}
& \frac{d \xi}{d t}=-\frac{\left(\frac{m c}{e g}\right)\left(V^{2}-0.5 V_{\perp}^{2}\right) \sin (\theta)}{A+\frac{\partial R_{0}}{\partial \xi} \cos (\theta)} \\
& \frac{d \theta}{d t}=\frac{\left(\frac{R c}{g}\right) \frac{\partial \Phi}{\partial \xi}+\left(\frac{V_{l}}{g}\right) \frac{\partial \Psi_{P}}{\partial \xi}-\left(\frac{m c}{e g}\right)\left(V^{2}-0.5 V_{\perp}^{2}\right) \frac{\partial R}{\partial \xi}}{A \xi\left(A+\frac{\partial R_{0}}{\delta \xi} \cos (\theta)\right)}
\end{aligned}
$$

and

$$
\frac{d V_{\|}}{d t}=\frac{\left(-\left(\frac{\mu}{m R^{2}}\right) \frac{\partial \Psi_{\rho}}{\partial \xi}+\left(\frac{c V_{\|}}{g}\right) \frac{\partial \Phi}{\partial \xi}\right) \sin (\theta)}{A+\frac{\partial R_{0}}{\partial \xi} \cos (\theta)}
$$




\section{Acknowledgements}

The authors would like to thank the following researchers: Dr. A. H. Boozer and Dr. R. B. White for insight into efficient and effective representation of the magnetic field in magnetic coordinates and for help in making the drift orbit equations Hamiltonian, Dr. S. P. Hirshman for providing an optimum definition of the poloidal angle $\theta$ which minimizes the number of Fourier moments needed to represent the magnetic field and for providing two independent methods for solving for the magnetic equilibium, Dr. R. M. Wieland for installing both methods in TRANSP, Dr. M. C. Zarnstorff for suggesting useful generalizations including plasma rotation, and Dr. S. M. have for providing challenging PBX (bean shaped) equilibria with which to test our vacuum region magnetic field representation. This work was supported by U.S. Department of Energy Contract Number DE-AC02-i6-CHO-3073.

\section{References}

1. R. B. White and M. S. Chance, "Hamiltonian Guiding Center Drift Orbit Calculation for Toroidal Plasmas of Arbitrary Cross Section," Phys. Fluids, 27, (1984).

2. A. H. Boozer, "Plasma Confinement," PPPL-2198, March 1985.

3. S. P. Hirshman and J. C. Whitson, Phys. Fluids, 26, 35.53 (1983).

4. S. P. Mirshman and H. K. Meier, Phys. Fluids, 28, 1387 (1985).

5. S. P. Hirshman and D. K. Lee, Comp. Phys. Comm., 39, 161 (1986).

6. L. L. Lao, et al, Comp. Phys. Comm., 27, 129 (1982). 